\title{
Economic Evaluation of Conventional Radiography with Film and Computed Radiography: Applied at BMC
}

\author{
Usama Ali Rahoma, Pavan Kumar Chundi \\ Department of Radiology, Faculty of Medical Technology, Omar Al-Mukthar University, Tobruk, Libya \\ Email: UsamaAliRahoma@yahoo.com,pavan9000@yahoo.com
}

Received September 16, 2012; revised October 26, 2012; accepted November 7, 2012

\begin{abstract}
Conventional radiography with film (CRF) has been in use for diagnostic purposes for a long time now. It has proved to be a great assert for the radiographers in assessing various abnormalities. With recent advances in technology it is now possible to have digital solutions for radiography problems at a very cost effective, environment friendly and also with better image quality in certain applications when compared to CRF. Rather than using a CRF a computed radiography (CR) uses imaging plates to capture the image. The imaging plate contains photosensitive phosphors which contain the latent image. Later this plate is introduced into a reader which is then converted into a digital image. The major advantage and the cost effective element of this system is the ability to reuse the imaging plates unlike the photographic film where in only a single image can be captured and cannot be reused. The computed radiography drastically reduces the cost by eliminating the use of chemicals like film developers and fixers and also the need for a storage room. It also helps to reduce the costs that are involved in the disposal of wastes due to conventional radiography. This paper investigates whether it is cost effective to use computed radiography over film based system at Al-Batnan Medical Center (BMC), Tobruk, Libya by using Cost Benefit Analysis (CBA). Apart from the initial cost of the CR System, based on the data collected from the center, from the year 2008 to 2012 (until June 2012) a total of 581,566 images were produced with the total cost incurred using film based system being USD 4,652,528. If the same number of images were produced using a CR system the total cost incurred would have been USD 82,600. Taking into consideration the cost of a new CR system to be USD 120,000 the overall cost of producing these images is USD 202,600. It is observed that an amount of USD 4,449,928 could have been saved over the period of 5 years starting from 2008 to 2012 by using the CR system at BMC. Using Cost Benefit Analysis, the average value of the net difference between the costs and benefits for the conventional film based system is -83.38 where as for the Computed System it is 22.06. Based on the principles of Cost Benefit Analysis it can be concluded that the system with a net positive difference is more cost beneficial than the other. With the help of the above two analysis it can be concluded that the use of computed radiography is definitely more cost effective for use at $\mathrm{BMC}$, when compared to the conventional x-ray radiography.
\end{abstract}

Keywords: Radiographic Film; X-Ray Radiography; Computed Radiography; Cost Benefit Analysis

\section{Introduction}

Computed radiography (CR) is a digital imaging technology and digital $\mathrm{x}$-ray image management system that has been used in human and veterinary medicine since the 1980s. CR helps eliminate many of the disadvantages of traditional radiography. A storage phosphor plate collects the pattern of $x$-ray attenuation that is extracted by a reader, which converts the data into a digital signal. The image is stored like any computer file and can be viewed on a computer screen, transmitted electronically, or printed out on paper or transparent film, similar to x-ray film [1-3]. Computer software allows manipulation of the digital image to enhance viewing. Rather than utilizing conventional $\mathrm{x}$-ray film to capture an image, computed radiography uses an imaging plate. This plate contains photo sensitive storage phosphors which retain the latent image. When the imaging plate is scanned with a laser beam in the digitizer, the latent image information is released as visible light $[1,4,5]$. The imaging plate is a flexible image sensor in which bunches of very small crystals (grain size about $5 \mu \mathrm{m}$ to 25 microns) of photostimulable phosphor [5]. The first step involved in this process is the exposure of the phosphor plate to record an image. The image thus recorded here is an invisible latent image. Now the phosphor plate is made to pass through a reader and processing unit. In this unit the screen is scanned by a very small laser beam. When the laser beam strikes a screen it causes light to be produced. The light that is produced is proportional to the $\mathrm{x}$-ray exposure. The result is that an image in the form of light is produced on the surface of the phosphor screen. As the 
surface of the phosphor screen is scanned by the laser beam, the analog data representing the brightness of the light is converted into digital values for each pixel and stored in the computer memory as a digital image [6-8]. The CR system also eliminates the need of a dark room and also the disposal of the chemical wastes that are produced during the development of the film $[9,10]$.

\subsection{Cost Benefit Analysis}

Cost benefit analysis is a basic tool that can be utilized to improve the decision making process in the allocation of funds to health and other. It is a method of comparing costs and benefits of alternative programs which are competing for funds and resources. It is recommended that cost-benefit analysis be used as a mechanism to evaluate innovative health programs. This may be the sole mechanism that will enable health practitioners to cost-justify their innovative services to the federal government and other third-party payers [11]. There are specific measurements for the viability of health services in treating diseases and illness which is called economic evaluation. These are important techniques and tools for health economics to evaluate the health outcomes by studying the medical cost, profit and benefits taken from the patients' health. There are four (4) forms of economic evaluation which are used predominately in public health; these are Cost Analysis (CA), Cost Effective Analysis (CEA), Cost Utility Analysis (CUA) and Cost Benefit Analysis (CBA). This is to ensure the economic feasibility, acceptability or viability in deciding to implement a specific health program whether it is in public sector or private sector. It should be noted that the most common profitability indicators being used to come up with feasibility study of the private sector in the establishment of hospitals, clinics and medical centers is the Cost Benefit Analysis. This is to ensure that their investments (cost of doing the project) are highly feasible. The return of investments are sustained at given supply price such as the user rate of hospital services, laboratory examinations, professional fees of health personnel and other hospital charges. The economic evaluations on cost utility analysis are being used to decide the economic acceptability of the project to support the prevention, promotion and rehabilitation of particular illnesses or diseases for social welfare. The health services and programs are implemented to purchase the needed medical supplies, equipment and facilities including the professional fees of health personnel to specific target clients. The public health sector has to implement health services and programs such as vaccines for communicable and noncommunicable diseases, the use of contraceptives for the reproductive health, cancer screening, spray of DDT and etc. These examples on the implementation of health programs have direct, indirect and intangible benefits such as reduction in health risk, pain and suffering which cannot be estimated from market data.

\subsection{Advantages}

Many studies have stated numerous advantages of using a CR system with respect to image quality and also eliminating the need for retakes that result from over and under exposure $[2,4,6,7,12]$. Because $\mathrm{x}$-ray film has a limited linear response, a relatively small, under or over exposure may result in an unacceptable image [8]. With the help of CR system images that are too dark or light can be adjusted with the image management software thus eliminating the need for a retake. The other distinct advantage of the CR system is the possibility to view bone-only and soft-tissue only images from a single exposure [13]. In conventional $x$-ray film radiography, a numbers of images have to be taken to see both soft tissue and bone in detail. This can be done in a single image with the help of a CR system.

\subsection{Disadvantages}

The major disadvantages of using CR system include the initial cost, need for training and making the necessary changes for the new system. One important aspect that has to be taken into account before purchase is the ease of use of the new system. Depending on the user's computer skills, manipulation of images and use of other software can be a time consuming process [1]. The prices of CR systems are falling consistently and they are recommended for use in high volume practices. The initial cost of the CR system must be weighed against the benefits of using less film. Digital manipulation cannot make all images useful. Like other computer systems the images must be backed up. Another disadvantage can be the over processing of the image which can create artifacts. Optimal viewing of the digital images depends on the quality of the monitors being used and also the quality of the paper used to take print out. These can increase the cost of the CR system. Improper labeling or misidentification of the patient can make the image retrieval difficult. CR system cannot compensate for improper use of the system or poor staff training. This paper investigates whether the Economic Evaluation of conventional radiography with film and computed radiography: applied using cost benefit analysis (CBA).

\section{Material and Method}

Al-Batnan Medical Center in Tobruk, Libya is a multispecialty hospital catering to the needs of the people in and around Tobruk. The center's Radiology department provides various diagnostic services using conventional x-ray film techniques, computed tomography, NMR-Magnetic Resonance Imaging, Ultrasound scanning. From the 
data collected for the year 2008 there were a total of 100,160 cases that used various diagnostic techniques and in the year 2012 (until June) there were about 160,740 cases. Taking into account the increase in the number of cases using the various diagnostic techniques, the cost of developing films with conventional x-ray film radiography also increases. The Radiology Department at Al-Batnan Medical Center s equipped with a Konica Regius Nano CR system which is a single bay reader capable of processing 76 plates $(14 \times 17$ ") per hour [14]. The center has also a PCR-Eleva reader system which uses the Eleva work spot technology to give a clear and excellent image quality. A cost benefit analysis has been done with data collected from Al-Batnan Medical Center. The analysis is based on a model that was used to determine the cost benefit analysis of automated testing with IBM Rational Robot [15].

A key consideration in the use of flexible storage phosphor plates and CR systems is that any exposure source that can be used with conventional $\mathrm{x}$-ray films can also be used with this filmless technology. Also the flexible storage phosphor imaging plates can be directly substituted for film. This compatibility with existing sources and cassettes makes a transition from conventional film radiography to CR a fairly uncomplicated and inexpensive proposition. The most important aspect of the use of imaging plates is the ability to reuse them. Theoretically each imaging plate can be used thousands of times [16]. But keeping in view the improper handling of imaging plates can cause damage to the plates. Hence for the study, it is assumed that each imaging plate can be reused up to 5000 times. Thus the recurring costs can be drastically reduced by using the imaging plates. This also eliminates the need of chemicals like developer and fixer that are used in order to produce an image with conventional film radiography. Performing a cost benefit analysis involves the following steps i.e. identifying the costs and benefits. For the study the following costs for conventional and computed radiography systems were identified.

1) film (total cost for the no. of films used);

2) developer chemical costs;

3) silver recovery system;

4) chemical waste disposal;

5) film archiving (space + labour);

6) administration (film indexing);

7) Software + back-up system;

8) Cost of imaging plates;

9) Consumables (DVDs).

Benefits:

1) Image quality;

2) View bone-only and soft tissue only images;

3) No retakes (over and under exposure);

4) Artifacts (over processing);
5) Compatibility to PACS;

6) Ease of producing images.

After the benefits have been identified, we have to establish performance measures for each benefit and estimate the value of the benefit. If a benefit can't reasonably be assigned a monetary value, it should be valued using a more subjective, qualitative rating system (which assigns relative numerical values). Table 1 has been used to rate the benefits [15]. When intangible benefits are significant factors in the analysis, the dollar values of the tangible benefits can be converted to the same rating scale as used to arrive at the relative values of the intangible benefits. This is be done by dividing all dollar values by some arbitrary number, such as 10,000 , that will give values lower than the highest value on the "Level of Benefit" scale [15]. The costs involved in each system have been calculated in the following way. All the data that was collected is in Libyan Dinars (LYD). A conversion rate of $1 \mathrm{USD}=1.26 \mathrm{LYD}$ has been used to change the data in term of USD (\$).

1) film (total cost for the no. of films used ) $\$ 8$ per film;

2) developer chemical costs $\$ 50$ per unit per week. units per week are 52;

3) silver recovery system $\$ 40 \times 10$ hrs per week;

4) chemical waste disposal $\$ 60 \times 60$ per week;

5) film archiving (space + labour) $\$ 15,375$ per year;

6) administration (film indexing, 17 staff) $\$ 40 \times 17$ per week;

7) administration (on pc, 10 staff) $\$ 40 \times 10$ per week;

8) Cost of imaging plates $\$ 700$ per plate;

9) Consumables (DVDs) 0.25 LYD per DVD equivalent to $\$ 0.19$.

\section{Results}

The data pertaining to the number of films that were used was collected from the Emergency and Radiology department for the years starting from 2008 to June 2012. Cost for 100 films is 1000 Libyan Dinars (LYD) equivalent to 793 US Dollars. The conversion used here for the calculation is $1 \mathrm{USD}=1.26 \mathrm{LYD}$. Approximately 1 film costs 8 USD. Cost for 100,160 films is 801,280 USD for the

Table 1. Level of Benefits scale.

\begin{tabular}{cc}
\hline Level of benefit & Value \\
\hline Very high & 5 \\
High & 4 \\
Medium & 3 \\
Low & 2 \\
Very low & 1 \\
\hline
\end{tabular}


year 2008 (Table 2). Cost of one imaging plate is 700 USD which can be used for 5000 times. So for 100,160 images the number of imaging plates required is 20 . The total cost involved for 100,160 images is 14,000 USD (Table 2). From Tables 2 and 3, for the year 2008, it can be seen that the use of imaging plates and computed radiography system is more cost effective when compared to the conventional x-ray film radiography. Similar conclusions can be stated from Tables 2 and 3, that for the years 2009-2012 respectively, the computed radiography system is cost effective for use at Al-Batnan Medical Center, Tobruk, Libya. Table 4, shows the total costs involved in developing the images for the years from 2008 to 2012 by conventional film radiography and computed radiography. Table 5 shows, the benefits on a relative scale. From Table 6, based on cost benefit analysis it is seen that the net benefit value is positive for Computed Radiography when compared to conventional $\mathrm{x}$-ray system. As discussed earlier a system which has a positive net benefit value is considered to cost beneficial over the other. Based on the above two analysis the computed radiography system is cost effective for use at Al-Batnan Medical Center. However it should be noted that the benefits were evaluated on a scale which is subjective.

\section{Discussion}

If the study is extended to BMC in Libya it can be stated that huge amount money can be saved by using CR systems over a period of time. However this study has not taken into consideration the initial cost of equipment. The initial cost of a CR system (approximately 120,000 USD) is much higher than that of conventional $x$-ray film radiography (approximately 20,000 USD). It is important to note that the cost of CR systems is reducing and also the availability of the films has become less. Many studies have stated that apart from the recurring costs, the use of CR system can reduce the radiation dose, reduces the number of retakes, and eliminates the need for the use of chemicals and their disposal. Total No. of films used around 2008-2012 (up to June) are 581566 with total cost incurred using film based system is USD 4,652,528 where as the total cost incurred with a CR system is USD 82,600 . We found that the film based system when used has cost $\mathrm{BMC}$ more money when compared to the $\mathrm{CR}$ system.

Table 4 shows the total costs involved in producing images by both film based system and computed radiography system. Starting from 2008 to 2012 for each year all the costs have been calculated based on the data that was collected at BMC. It is observed that the costs

Table 2. Total cost and the No. of films used in the Emergency and Radiology Department for the year starting from 2008 to June 2012.

\begin{tabular}{ccc}
\hline Year & No. of films used & Total cost USD \\
\hline 2008 & 100160 & 801,280 \\
2009 & 101346 & 810,768 \\
2010 & 102580 & 820,640 \\
2011 & 116740 & 933,920 \\
2012 (up to June) & 160740 & $1,285,920$ \\
Total & 581566 & $4,652,528$ \\
\hline
\end{tabular}

Cost of 1 film $=8$ USD.

Table 3. Estimated cost by using imaging plates. Calculations are based on the No. films used in the Emergency and Radiology Department for the year starting from 2008 to June 2012.

\begin{tabular}{cccc}
\hline Year & No. of films used to develop images & Total no. of imaging plates required & Total cost (in USD) \\
\hline 2008 & 100160 & 20 & 14,000 \\
2009 & 101346 & 21 & 14,700 \\
2010 & 102580 & 21 & 14,700 \\
2011 & 116740 & 24 & 16,800 \\
2012 (up to June) & 160740 & 32 & 22,400 \\
Total & 581566 & 118 & 82,600 \\
\hline
\end{tabular}

Cost of 1 imaging plate $=700$ USD. 
Table 4. Indicating the total costs involved in developing the images for the years from 2008 to 2012 by conventional film radiography and computed radiography.

\begin{tabular}{|c|c|c|c|c|c|c|c|c|c|c|}
\hline \multirow{2}{*}{ Cost (in USD) } & \multicolumn{2}{|c|}{2008} & \multicolumn{2}{|c|}{2009} & \multicolumn{2}{|c|}{2010} & \multicolumn{2}{|c|}{2011} & \multicolumn{2}{|c|}{2012} \\
\hline & FR & CR & FR & CR & FR & CR & FR & CR & FR & CR \\
\hline Total cost for films & 801,280 & 0 & 810,768 & 0 & 820,640 & 0 & 933,920 & 0 & $1,285,920$ & 0 \\
\hline Developer chemical costs & 2600 & 0 & 2600 & 0 & 2600 & 0 & 2600 & 0 & 2600 & 0 \\
\hline Silver recovery system & 20,800 & 0 & 20,800 & 0 & 20,800 & 0 & 20,800 & 0 & 20,800 & 0 \\
\hline Chemical waste disposal & 3600 & 0 & 3600 & o & 3600 & 0 & 3600 & 0 & 3600 & 0 \\
\hline Film archiving (space + labour) & 15,375 & 0 & 15,375 & 0 & 15,375 & 0 & 15,375 & 0 & 15,375 & 0 \\
\hline Administration (film indexing) & 35,360 & 20,800 & 35,360 & 20,800 & 35,360 & 20,800 & 35,360 & 20,800 & 35,360 & 20,800 \\
\hline Software + back-up system & 0 & 25,000 & 0 & 2000 & 0 & 2000 & 0 & 2000 & 0 & 2000 \\
\hline Cost of imaging plates & 0 & 14,000 & 0 & 14,700 & 0 & 14,700 & 0 & 16,800 & 0 & 22,400 \\
\hline Consumables (DVDs) & 0 & 19,873 & 0 & 20,108 & 0 & 20,353 & 0 & 23,162 & 0 & 31,892 \\
\hline Total cost (USD) & 879,015 & 79,673 & 888,530 & 57,608 & 898,375 & 57,853 & $1,011,655$ & 62,762 & $1,363,655$ & 77,092 \\
\hline
\end{tabular}

Film radiography $=\mathrm{FR}$, Computed radiography $=\mathrm{CR}$.

Table 5. Indicating the benefits on a relative scale.

\begin{tabular}{ccc}
\hline Benefits & Film radiography & Computed radiography \\
\hline Image quality & 2 & 5 \\
View bone-only and soft only images & 1 & 4 \\
No retakes (over and under exposure) & 1 & 4 \\
Artifacts (over processing) & 4 & 2 \\
Compatibility to PACS & 1 & 5 \\
Ease of producing images & 2 & 4 \\
Electronic archiving for future reference & 1 & 4 \\
Lower dosage of x-rays to achieve the same quality & 2 & 4 \\
Eliminating the use of chemicals & 1 & 4 \\
Eliminating the need for chemical waste disposal & 1 & 4 \\
Reduction of storage space and manual labour & 1 & 45 \\
\hline
\end{tabular}

Table 6. Converting cost into equivalent numbers (cost/ 10,000).

\begin{tabular}{ccc}
\hline Year & Film based radiography & Computed radiography \\
\hline 2008 & 87.9 & 7.9 \\
2009 & 88.8 & 5.7 \\
2010 & 89.8 & 5.7 \\
2011 & 101.1 & 6.2 \\
2012 & 136.3 & 7.7 \\
\hline
\end{tabular}

involved in producing images by film based system are much higher when compared to the costs involved using computed system. Over the years the number of images taken has increased and thereby the operational costs involved have also increased. The high operational costs involved in the film based system are due to the cost of the films, use of chemicals to develop the images, disposal of chemicals, cost of administration staff for film indexing and storage. All these costs over a period of time have the film based system a costly affair. While coming to the computed radiography system, in the year 2008 , the operational costs are high due to initial cost 
involved in purchasing scanner and recovery software. In the following years the CR system has seen low operational costs when compared to the film based system. All the costs involving chemicals and disposal, use of additional administrative staff for film indexing and storage have been completely eliminated. Instead of using a 17 member team for film indexing a CR system uses just 10 staff for administration on PC thereby reducing the operational costs.

Keeping in view the use of film based system and computed system the general benefits have been listed in Table 5. A relative measure on a scale of 1 - 5 has been given to each benefit and the total obtained for each system has been calculated. The above benefits are called as intangible benefits where we cannot assign a monetary value for these benefits. But these benefits play a significant role in the economic evaluation of the systems. Based on the previous studies involving film based system and CR system the above benefits are listed to be the most significant ones that play a key role in making a decision. From the above table it can be seen that for the benefit eliminating the use of chemicals, under film based system it has value of 1 where as under CR system it has 4. This is because in film based system we cannot eliminate the use of chemicals but in case of CR system this possible so value of 4 has been assigned under it. Similarly for the benefit artifacts (over processing) it can be seen that the under film based system it has a value of 4 where as under CR system it is 2 . This is because of the possibility of over processing and manipulation of the image with the CR system which can produce artifacts there by creating undesirable images which can lead to wrong diagnosis. But this is not possible with a film based system as it doesn't involve any software to develop the images. So a value of 4 has been assigned under it. Similarly, each benefit has been valuated with reference to both the system and a relative reference value has been assigned under each system.

Cost benefit analysis is being used in this study to evaluate the two systems. As seen before we need to have a comparable scale to compare the costs and benefits. It is seen that the costs involved in the study are in term of dollars where as the benefits have been assigned a relative numerical value based on the level of benefits scale. In order to compare the costs and benefits and to arrive to a conclusion it is highly desirable to have the costs and benefits on the same scale i.e. either have both costs and benefits in terms of dollars or both expressed on a relative scale. Since the benefits in this study are intangible benefits it is very difficult to assign a dollar value to each benefit. Hence the costs have been converted to a relative scale in order to compare with the benefits. To achieve this, the total costs involved in each year for the two systems have been divided by an arbitrary number 10,000 , that gave values lower than the highest value on the "Level of Benefit" scale. Now the costs and benefits are on a comparable scale and a cost benefit analysis can be performed to evaluate each system. Tables 6 and 7 show the results obtained after converting the costs for each year for both the system involved in the study and cost benefit difference. A system that has a net positive difference is considered to be cost beneficial over the other. For the year 2008 the film based system has a difference of -70.9 and the CR system -43.8 . The negative difference for the CR system is due to initial cost of the scanner and software backup system. In the year 2009 the difference for a film based system is -71 and the CR system is 39. This trend is observed for all the years from 2009 to 2012 . The CR system has a net positive difference indicating that the system has an overall net cost benefit when compared to the film based system. This analysis can be useful tool for decision making whether to go for the new system or not. Based on the results seen above we can find that the use of CR system at BMC is cost beneficial when compared to the film based system.

Over the years it can be observed that the net difference value which is negative for film based system has increased from -70.9 in the year 2008 to -119 in the year 2012. This increase in difference is due to the increase in the number of images taken and thereby an overall increase in the cost to produce and store such images. The cost benefit analysis has been a useful tool in the field of medicine and healthcare especially in designing and implementing healthcare programs which involve high costs. The important factor to be noted while performing a cost benefit analysis is that the measure of level of benefits in highly subjective and may vary from person to person. However based on the data and previous studies involving CR system and film based

Table 7. Cost benefit difference table.

\begin{tabular}{|c|c|c|c|c|c|c|c|c|c|c|c|c|c|c|c|}
\hline \multirow{2}{*}{ System } & \multicolumn{3}{|c|}{2008} & \multicolumn{3}{|c|}{2009} & \multicolumn{3}{|c|}{2010} & \multicolumn{3}{|c|}{2011} & \multicolumn{3}{|c|}{2012} \\
\hline & $\mathrm{C}$ & $\mathrm{B}$ & $\mathrm{D}$ & $\mathrm{C}$ & B & $\mathrm{D}$ & $\mathrm{C}$ & B & $\mathrm{D}$ & $\mathrm{C}$ & B & $\mathrm{D}$ & $\mathrm{C}$ & B & $\mathrm{D}$ \\
\hline FB & 87.9 & 17 & -70.9 & 88.8 & 17 & -71 & 89.8 & 17 & -72 & 101 & 17 & -84 & 136 & 17 & -119 \\
\hline Com & 88.8 & 45 & -43.8 & 5.7 & 45 & 39 & 5.7 & 45 & 39 & 6.2 & 45 & 38.8 & 7.7 & 45 & 37.3 \\
\hline
\end{tabular}

$\mathrm{FB}=$ Film based, $\mathrm{Com}=$ computed, $\mathrm{C}=$ cost, $\mathrm{B}=$ Benefit, $\mathrm{D}=$ Diff. 
system, it can be concluded that the use of CR system is cost beneficial when compared to film based system.

\section{Conclusion}

For the years starting from 2008 to 2012, the average value of the net difference between the costs and benefits for the conventional film based system is -83.38 where as for the CR System it is 22.06. Based on the principles of Cost Benefit Analysis it can be concluded that the system with a net positive difference is more cost beneficial than the other. Apart from the initial cost of the CR System, based on the data collected from the center a total of 581566 images were produced with the total cost incurred using film based system being USD 4,652,528. If the same number of images were produced using a CR system the total cost incurred would have been USD 82,600. Taking into consideration the cost of a new CR system to be USD 120,000 , the overall cost for producing the images is USD 202,600. It is observed that an amount of USD 4,449,928 could have been saved over the period of 5 years starting from 2008 to 2012 (up to June) by using the CR system at BMC. With the help of the above two analysis it can be concluded that the use of computed radiography is definitely more cost effective for use at $\mathrm{BMC}$, when compared to the conventional $\mathrm{x}$-ray radiography. Considering the fact that center's staff is well trained in using the CR system, it is economical to use this system with respect to the operational and recurring costs when compared to the conventional x-ray film radiography.

\section{REFERENCES}

[1] G. Roberts and J. Graham, "Computed Radiography," In: S. Kraft and G. Roberts, Eds., Modern Diagnostic Imaging, Veterinary Clinics of North America: Equine Practice, Saunders, Philadelphia, 2001, pp. 47-62.

[2] R. G. Swee, J. E. Gray and J. W. Beabout, "Screen-Film versus Computed Radiography Imaging of the Hand: A Direct Comparison," American Journal of Roentgenology, Vol. 168, No. 2, 1997, pp. 539-542.

[3] S. Don, C. F. Hildebolt and T. L. Sharp, "Computed Radiography versus Screen Film Radiography: Detection of Pulmonary Edema in a Rabbit Model that Stimulates Neonatal Pulmonary Infiltrates," Radiology, Vol. 213, 1999, pp. 455-460.
[4] R. E. Greene and J. Oestmann, "Computed Digital Radiography in Clinical Practice," Thieme Medical Publishers, New York, 1992, pp. 2-46.

[5] J. R. Patel, "Digital Applications of Radiography," 3rd Middle East Nondestructive Testing Conference \& Exhibition, Manama, 27-30 November 2005, pp. 27-30.

[6] B. Reiner, E. Siegel, T. Mc Laurin, et al., "Evaluation of Soft-Tissue Foreign Bodies: Comparing Conventional Plain Film Radiography, Computed Radiography Printed on Film, and Computed Radiography Displayed on A Computer Workstation," American Journal of Roentgenology, Vol. 167, No. 1, 1996, pp. 141-144.

[7] S. A. Wegryn, D. W. Piraino, B. J. Richmond, et al., "Comparison of Digital and Conventional Musculoskeletal Radiography: An Observer Performance Study," Radiology, Vol. 175, No. 1, 1990, pp. 1225-1228.

[8] G. Roberts, "Computed radiography: How It works and Its Advantages," The AAEP 2000 Resort Symposium Lecture Workbook, 4-6 February 2000.

[9] P. J. Lund, E. A. Krupinski, S. Pereles and B. Mockbee, "Comparison of Conventional and Computed Radiography: Assessment of Image Quality and Reader Performance in Skeletal Extremity Trauma," Academic Radiology, Vol. 4, No. 8, 1997, pp. 1570-576. doi:10.1016/S1076-6332(97)80207-3

[10] M. Ogoda, "DICOM 101: Understanding the Basics of DICOM. Insights \& Images," The User's Publication of Computed Radiography, Fujifilm Medical Systems, Stamford, 2001, pp. 2-4.

[11] J. L. Bootman, C. Rowland and A. I. Wertheimer, "CostBenefit Analysis: A Research Tool for Evaluating Innovative Health Programs," Evaluation \& the Health Professions, Vol. 2, No. 2, 1979, pp. 129-154. doi: $10.1177 / 016327877900200202$

[12] Z. F. Lu, E. L. Nickoloff, J. C. So and A. K. Dutta, "Comparison of Computed Radiography and Film Screen Combination Using a Contrast-Detail Phantom," Journal of Applied Clinical Medical Physics, Vol. 4, No. 1, 2003, pp. 91-98. doi:10.1120/1.1524950

[13] Fujifilm, "Advanced Processing Capabilities of FCR," 2003.

[14] Konica Minolta, "Nano CR Clinic Brochure," 2007.

[15] K. Micheal, "Using Cost-Benefit Analysis to Compare Different Test Structures for Rational Robot," 19 November 2003.

[16] Siemens Medical, "Computed Radiography System. An Easy Step from Analog to Digital,” 2006. 